\title{
Combined Differential and Linear Cryptanalysis of Reduced-Round PRINTCIPHER
}

\author{
Ferhat Karakoç ${ }^{1,2}$, Hüseyin Demirci ${ }^{1}$, and A. Emre Harmanci ${ }^{2}$ \\ 1 Tübitak BILGEM UEKAE, 41470, Gebze, Kocaeli, Turkey \\ \{ferhatk, huseyind\}@uekae.tubitak.gov.tr \\ ${ }^{2}$ Istanbul Technical University, Computer Engineering Department, 34469, Maslak, \\ Istanbul, Turkey \\ harmanci@itu.edu.tr
}

\begin{abstract}
In this paper we analyze the security of PRINTCIPHER using a technique that combines differential and linear cryptanalysis. This technique is different from differential-linear cryptanalysis. We use linear approximations to increase the probability of differential characteristics. We show that specific choices of some of the key bits give rise to a certain differential characteristic probability, which is far higher than the best characteristic probability claimed by the designers. We give the underlying mechanism of this probability increase. We have developed attacks on 29 and 31 rounds of PRINTCIPHER- 48 for $4.54 \%$ and $0.036 \%$ of the keys, respectively. Moreover, we have implemented the proposed attack algorithm on 20 rounds of the cipher.
\end{abstract}

Keywords: PRINTCIPHER, differential cryptanalysis, linear cryptanalysis, differential-linear cryptanalysis.

\section{Introduction}

The security and privacy in constrained environments such as RFID tags and sensor networks is a challenging subject in cryptography. Lightweight cryptographic algorithms and protocols are required for this reason. Some block and stream ciphers and hash functions are proposed to meet this requirement [2, 3, 8, 1012, 17, 18, 22, 27, 28]. The encryption algorithm PRINTCIPHER was introduced in CHES 2010 as a lightweight block cipher by Knudsen et al. [19]. The authors aim to build an algorithm especially suitable for integrated circuit printing.

At FSE 2011, Abdelraheem et al. [1] applied a differential attack on reduced rounds of PRINTCIPHER. Their attack can break half of the rounds of the cipher. The authors have observed that the differential distribution has a key dependent structure. They have exploited this fact to get information about the key bits. Their attack uses the whole codebook and has a complexity about $2^{48}$ computational steps for the 48-bit version of the algorithm. The authors use the roots of permutations to deduce the key bits which affect the key-dependent permutations. There are also algebraic cryptanalysis and side channel analyses of PRINTCIPHER [9, 31]. But, the designers noticed that side channel and related key attacks were not their major concern in the design of PRINTCIPHER.

A. Miri and S. Vaudenay (Eds.): SAC 2011, LNCS 7118, pp. 169-184, 2012.

(C) Springer-Verlag Berlin Heidelberg 2012 
Recently, Leander et al. [21] have announced an attack on the full round PRINTCIPHER-48 for a class of $2^{52}$ keys. Also Agren et al. [15] have applied a linear attack on 28-round PRINTCIPHER-48 which works for half of the keys.

Differential [7] and linear cryptanalysis [25] are the most used cryptanalysis techniques for block ciphers. Also there are some attacks which uses combinations of classical techniques such as impossible-differential [4], boomerang [30], and differential-linear attack [20]. In the differential-linear method, the attacker divides the cipher into two parts where a differential and a linear approximation are constructed for the first and second parts respectively. This combined attack method was enhanced by some other works [5, 23, 32] and applied on some ciphers such as IDEA and Serpent [6, 14, 16]. Also, there are some key-dependent attacks [13, 16, 26, 29] where [16] uses a differential-linear technique.

In this work, we combine differential and linear cryptanalysis in a different technique on PRINTCIPHER. We construct linear approximations to increase the probability of differential characteristics. Using this method we have found that for some of the keys, the probability of an $r$-round differential characteristic is significantly higher than the maximum probability of $r$-round characteristic claimed by the designers. We point out the special key values which induce to this weakness and explain the mechanism behind this observation. We show that $4.54 \%$ and $0.036 \%$ of the keys are weak for 29 and 31 rounds, respectively.

This paper proceeds as follows. In Section 2, we briefly introduce the notation we use and the PRINTCIPHER. In Section 3, we explain the weak key mechanism of the cipher. Section 4 is on the cryptanalytic attacks using the observations of the previous section. Finally, we conclude the paper in Section 5.

\section{The PRINTCIPHER Encryption Algorithm}

\subsection{Notation}

Throughout this paper, we use $s k_{1}$ for the key used in the xor part and $s k_{2}$ for the key which is used to determine the key dependent permutation. The letters $x^{i}, y^{i}, z^{i}, t^{i}$ denote the inputs of the round, the permutation, the key dependent permutation and the S-box layers in the $i$-th round, respectively. $x[i]$ represents the $i$-th bit of the variable $x$ where $x[0]$ is the rightmost bit of $x$. Also $x[i-j]$ is the bit string $x[i] x[i-1] \ldots x[j]$, where $i>j$. We denote the number of 1 's of a bit vector by $h w\left(x_{i}, \ldots, x_{1}, x_{0}\right)$. We write the bit values between parentheses. Also we use $\Delta$ to indicate the difference between two bit strings.

\subsection{PRINTCIPHER}

The PRINTCIPHER encryption algorithm has two versions, PRINTCIPHER-48 has block size of 48 bits, consists of 48 rounds and uses 80-bit key whereas PRINTCIPHER-96 has block size of 96 bits, consists of 96 rounds and admits 160-bit key.

PRINTCIPHER has an SP-network structure where the S-box is chosen to have the best differential and linear distributions among 3-bit functions. Each 
round function consists of a key xoring, a bitwise permutation over 48 (resp 96) bits, a round-constant xoring to the least significant bits, a bitwise permutation on 3 bits and an S-box layer.

Note that the round key $k=s k_{1} \| s k_{2}$ is identical at each round. The first $b$ bits of the key, $s k_{1}$, is xored to the state at the beginning of each round. After that the following bit permutation is applied:

$$
P(i)= \begin{cases}3 i \bmod b-1 & \text { for } 0 \leq i \leq b-2, \\ b-1 & \text { for } i=b-1\end{cases}
$$

where $b \in\{48,96\}$ is the block size. Then, a 6 -bit or a 7 -bit round constant is added to the least significant bits of the state according to the block size. We would like to point out that most significant bits are not affected from this addition. This is followed by a key dependent permutation. In this layer, $s k_{2}$ is divided into 2-bits and each 2-bit is used to determine the permutation on each 3 -bit of the state. This permutation is defined as follows where $a_{1} \| a_{0}$ are the bits of $s k_{2}$ and $c_{2}\left\|c_{1}\right\| c_{0}$ are the state bits:

\begin{tabular}{cc}
$a_{1} \| a_{0}$ & \\
\hline 00 & $c_{2}\left\|c_{1}\right\| c_{0}$ \\
01 & $c_{1}\left\|c_{2}\right\| c_{0}$ \\
10 & $c_{2}\left\|c_{0}\right\| c_{1}$ \\
11 & $c_{0}\left\|c_{1}\right\| c_{2}$
\end{tabular}

Finally, the same S-box is applied in parallel to every 3-bit of the state. The unique S-box used in PRINTCIPHER is the following:

\begin{tabular}{|c||c|c|c|c|c|c|c|c|}
\hline$x$ & 0 & 1 & 2 & 3 & 4 & 5 & 6 & 7 \\
\hline$S[x]$ & 0 & 1 & 3 & 6 & 7 & 4 & 5 & 2 \\
\hline
\end{tabular}

The round function of PRINTCIPHER-48 is shown in Figure 1, For a more detailed description of PRINTCIPHER we refer to [19].

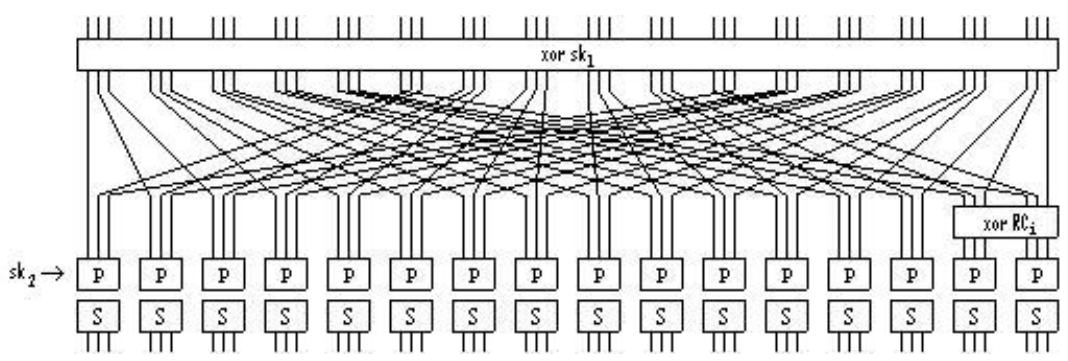

Fig. 1. One round of PRINTCIPHER-48 


\section{Key Dependent Differential and Linear Characteristics}

According to the designers of PRINTCIPHER, maximum probability of an $r$ round differential characteristic for both versions of the cipher is $2^{-2 \times r}$. However, we have found that an $r$-round differential characteristic for PRINTCIPHER-48 can have a probability of about $2^{-(6+1.68 \times(r-3))}$ for $4.54 \%$ of the keys and a probability of $2^{-(7.68+1.51 \times(r-4))}$ for $0.036 \%$ of the keys. To clarify the significance of the probabilities note that for 24-round differential characteristic we have a probability of $2^{-41.28}$ for the first and $2^{-37.88}$ for the second key subset. But according the designers of the algorithm the maximum probability of 24-round differential characteristic is $2^{-48}$. Also for PRINTCIPHER-96 one can find similar subsets of the keys resulting higher probabilities than the probability expressed by the designers. We will focus on the analysis of PRINTCIPHER-48 in this paper.

The reason of this probability increase is the correlation of the input and output bits of active S-boxes in the differential paths in consecutive rounds. To express the reason in detail first we give differential and linear properties of the S-box.

\subsection{Differential and Linear Properties of the S-Box}

The S-box conserves one bit input difference in the same position in the output with probability $2^{-2}$, see Table1.

Table 1. Difference distribution table of the S-box

\begin{tabular}{rr|c|c|c|c|c|c|c|c|} 
& & \multicolumn{7}{|c}{ Output Difference } \\
& & 000 & 001 & 010 & 011 & 100 & 101 & 110 & 111 \\
\hline 000 & 8 & 0 & 0 & 0 & 0 & 0 & 0 & 0 \\
001 & 0 & 2 & 0 & 2 & 0 & 2 & 0 & 2 \\
Input & 010 & 0 & 0 & 2 & 2 & 0 & 0 & 2 & 2 \\
Difference & 100 & 0 & 2 & 2 & 0 & 0 & 2 & 2 & 0 \\
& 101 & 0 & 2 & 0 & 2 & 2 & 0 & 2 & 0 \\
110 & 0 & 0 & 2 & 2 & 2 & 2 & 0 & 0 \\
111 & 0 & 2 & 2 & 0 & 2 & 0 & 0 & 2 \\
\hline
\end{tabular}

Similarly, it can be seen in Table 2 that $i$-th bit of the input of the S-box equals to $i$-th bit of the output with a bias $2^{-2}$ or $-2^{-2}$.

\subsection{Characteristics for $4.54 \%$ of the Keys of PRINTCIPHER-48}

Using the properties of the S-box mentioned in the previous section and putting some conditions on the key bits we are able to combine a differential and a linear characteristic resulting a differential characteristic with higher probability than 
Table 2. Linear approximation table of the S-box

\begin{tabular}{r|r|r|r|r|r|r|r|r|r|} 
& \multicolumn{7}{|c}{ Output Mask } \\
& 000 & 001 & 010 & 011 & 100 & 101 & 110 & 111 \\
\hline 000 & 4 & 0 & 0 & 0 & 0 & 0 & 0 & 0 \\
001 & 0 & -2 & 0 & 2 & 0 & 2 & 0 & 2 \\
010 & 0 & 0 & 2 & 2 & 0 & 0 & 2 & -2 \\
Input 011 & 0 & 2 & -2 & 0 & 0 & 2 & 2 & 0 \\
Mask 100 & 0 & 0 & 0 & 0 & 2 & -2 & 2 & 2 \\
101 & 0 & 2 & 0 & 2 & 2 & 0 & -2 & 0 \\
110 & 0 & 0 & 2 & -2 & 2 & 2 & 0 & 0 \\
111 & 0 & 2 & 2 & 0 & -2 & 0 & 0 & 2 \\
\hline
\end{tabular}

$2^{-2}$ for one round. We have found 3 different combined characteristics each of which puts 6-bit conditions on the key bits of PRINTCIPHER-48. One of the characteristics is shown in Figure 2 and the other characteristics are given in Figure 6 and Figure 7 in Appendix [A. To express the reason of the probability increase we focus on the characteristic shown in Figure 2 . The probability increase for the other characteristics depends on a similar reason.

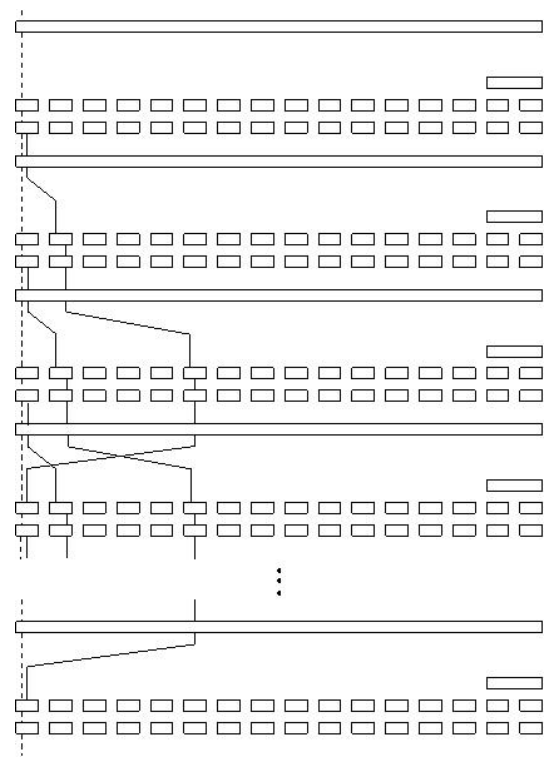

Fig. 2. Characteristic 1: A combined differential and linear characteristic which has probability $2^{-(6+1.68 \times(r-3))}$ for $r$ rounds

In Figure 2, the dotted line and the solid line shows the differential path and the linear path respectively. We give the following lemma to show the correlation of the input-output bits of the active S-boxes in the differential path in consecutive rounds using the linear path in Figure 2 
Lemma 1. Let the key bits of PRINTCIPHER-48 satisfy the following equations

$$
s k_{2}[29]=1, s k_{2}[28]=1, s k_{2}[21]=0, s k_{2}[20]=1 .
$$

Then, the bias of the equation $x^{i}[46] \oplus s k_{1}[46] \oplus s k_{1}[42] \oplus s k_{1}[31]=z^{i+2}[46]$ is $-2^{-3}$ where $x^{i}$ is the input of the $i$-th round and $z^{i+2}$ is the input of the key dependent permutation in the $(i+2)$-th round.

Proof. Let the three input bits of the S-box be $i_{2} i_{1} i_{0}$ and three output bits be $\mathrm{O}_{2} \mathrm{O}_{1} \mathrm{O}_{0}$. From the linear approximation table of the S-box, the biases of equations $i_{0} \oplus o_{0}=0$ and $i_{1} \oplus o_{1}=0$ are $-2^{-2}$ and $2^{-2}$ respectively. Using this information we can write the following equations with the corresponding biases:

$$
\begin{aligned}
& t^{i}[42]=x^{i+1}[42], \\
& t^{i+1}[31]=x^{i+2}[31], \epsilon=2^{-2} .
\end{aligned}
$$

Also using the following equations

$$
\begin{gathered}
x^{i}[46] \oplus s k_{1}[46]=t^{i}[42]\left(\text { since } s k_{2}[29]=1 \text { and } s k_{2}[28]=1\right) \\
x^{i+1}[42] \oplus s k_{1}[42]=t^{i+1}[31]\left(\text { since } s k_{2}[21]=0 \text { and } s k_{2}[20]=1\right) \\
x^{i+2}[31] \oplus s k_{1}[31]=z^{i+2}[46]
\end{gathered}
$$

we can reach the equation $x^{i}[46] \oplus s k_{1}[46] \oplus s k_{1}[42] \oplus s k_{1}[31]=z^{i+2}[46]$ with bias $2 \times\left(-2^{-2}\right) \times 2^{-2}=-2^{-3}$ using the Piling-up Lemma [25].

The correlation of the input and output bits of the active S-boxes in consecutive rounds helps us to give one of our main statements for the probability of the differential characteristic shown in Figure 2.

Theorem 1. Let the key bits of PRINTCIPHER-48 satisfy the following equations

$$
\begin{gathered}
s k_{2}[30]=0, s k_{2}[29]=1, s k_{2}[28]=1, s k_{2}[21]=0, s k_{2}[20]=1, \\
s k_{1}[46] \oplus s k_{1}[42] \oplus s k_{1}[31]=1 .
\end{gathered}
$$

Then, the probability of the differential characteristic $(100 \ldots 00) \rightarrow(100 \ldots 00) \rightarrow$ $\ldots \rightarrow(100 \ldots 00)$ for $r$ rounds is $2^{-(6+1.68 \times(r-3))}$.

Proof. Since $s k_{2}[30]=0$, the key dependent permutation layer keeps the difference in the leftmost bit. In the first three rounds, the probability of the differential characteristic is $2^{-6}$ because there is no linear relation between the input-output bits of the active S-boxes. In the fourth round, while $z^{4}[45]$ is distributed uniformly, $z^{4}[46]$ equals to $x^{2}[46] \oplus s k_{1}[46] \oplus s k_{1}[42] \oplus s k_{1}[31]$ with bias $-2^{-3}$ putting $i=2$ in Lemma 1 . We know that $x^{2}[46]=1$ because only the pair $(011,111)$ conserves the difference in the leftmost bit for the S-box and the corresponding output pair is $(110,010)$. Since $s k_{1}[46] \oplus s k_{1}[42] \oplus s k_{1}[31]=1$, we have $z^{4}[46]=1$ with bias $2^{-3}$, that is with probability $10 / 16$. Thus, for the fourth round the input pair of the S-box is $(011,111)$ with probability $2^{-1} \times 10 / 16=$ $2^{-1.68}$. That means the difference in the leftmost bit of the inputs of the S-box stays in the same position in the output of the S-box with probability $2^{-1.68}$. For the later rounds $z^{i}[46]$ equals to $x^{i-2}[46] \oplus s k_{1}[46] \oplus s k_{1}[42] \oplus s k_{1}[31]=1$ 
with probability $10 / 16$ and we may assume that $z^{i}[45]$ has a uniform distribution. That is, the probability for the rounds greater than four is $2^{-1.68}$. Thus the probability of the $r$-round differential characteristic is $2^{-(6+1.68 \times(r-3))}$.

In Table 3, the key constraints for the combined characteristics are shown. We use the notation $K S^{i}$ to show the key subsets which satisfy the $i$-th combined characteristic.

Table 3. Key constraints for the combined characteristics 1, 2, and 3

\begin{tabular}{c|c|c}
$\begin{array}{c}\text { Combined } \\
\text { Characteristic }\end{array}$ & $\begin{array}{c}\text { Key } \\
\text { Conditions }\end{array}$ & $\begin{array}{c}\text { Key } \\
\text { Subset }\end{array}$ \\
\hline Characteristic 1 & $s k_{2}[30]=0, s k_{2}[29]=1, s k_{2}[28]=1, s k_{2}[21]=0$, & $K S^{1}$ \\
& $s k_{2}[20]=1, s k_{1}[46] \oplus s k_{1}[42] \oplus s k_{1}[31]=1$ & \\
\hline Characteristic 2 & $s k_{2}[17]=1, s k_{2}[16]=0, s k_{2}[19]=0, s k_{2}[18]=1$, & $K S^{2}$ \\
& $s k_{2}[27] \oplus s k_{2}[26]=0, s k_{1}[40] \oplus s k_{1}[29] \oplus s k_{1}[25]=0$ & \\
\hline Characteristic 3 & $s k_{2}[15]=0, s k_{2}[14]=1, s k_{2}[13]=1, s k_{2}[12]=0$, & $K S^{3}$ \\
& $s k_{2}[5] \oplus s k_{2}[4]=0, s k_{1}[22] \oplus s k_{1}[18] \oplus s k_{1}[7]=1$ & \\
\hline
\end{tabular}

Analyzing the above conditions we have observed that the size of the key space which satisfies at least one of the constraints in Table 3 is $4.54 \%$ of the key space of the PRINTCIPHER-48.

\subsection{Characteristics for $\mathbf{0 . 0 3 6 \%}$ of the Keys of PRINTCIPHER- 48}

We increase the probability of $r$-round differential characteristic to $2^{-(7.68+1.51 \times(r-4))}$ for $0.036 \%$ of the keys putting extra conditions on the key bits of PRINTCIPHER- 48 .

In Section 3.2, we use only one linear characteristic to increase the probability of the differential characteristic. To proceed the increase of the probability of the differential characteristic we use an extra linear characteristic in this section. We have found two different combined characteristics which puts 12 and 13-bit conditions on the key bits of PRINTCIPHER-48 with the probability $2^{-(7.68+1.51 \times(r-4))}$ for $r$-round differential characteristic. One of the characteristics is shown in Figure 3 and the other characteristic is given in Figure 8 in Appendix $\mathrm{B}$. We give the reason of the probability increase for the first characteristic. The second can be derived by similar techniques.

The correlation of the bits in one of the linear paths is stated in Lemma 1 The following lemma states the correlation of the bits in the new linear path.

Lemma 2. Let the key bits of PRINTCIPHER-48 satisfy the following equations

$$
s k_{2}[10]=0, s k_{2}[27]=s k_{2}[26]=s k_{2}[15]=s k_{2}[14]=s k_{2}[11]=1 .
$$

Then, the bias of the equation $x^{i}[45] \oplus s k_{1}[45] \oplus s k_{1}[39] \oplus s k_{1}[21] \oplus s k_{1}[15]=$ $z^{i+3}[45]$ is $-2^{-4}$. 

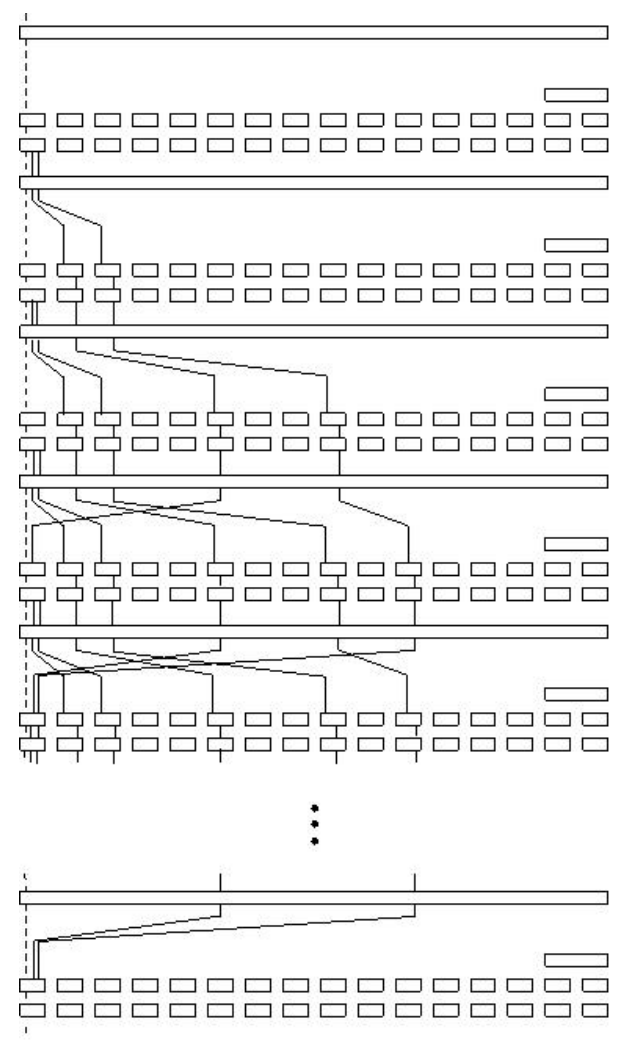

Fig. 3. Characteristic 4: A combined differential and linear characteristic which has probability $2^{-(7.68+1.51 \times(r-4))}$ for $r$ rounds

Proof. We can write the following equations with corresponding biases using linear approximation table of the S-box:

$$
\begin{gathered}
t^{i}[39]=x^{i+1}[39], \epsilon=-2^{-2} \\
t^{i+1}[21]=x^{i+2}[21], \epsilon=-2^{-2} \\
t^{i+2}[15]=x^{i+3}[15], \epsilon=-2^{-2} .
\end{gathered}
$$

Also using the following equations

$$
\begin{gathered}
x^{i}[45] \oplus s k_{1}[45]=t^{i}[39]\left(\text { since } s k_{2}[27]=1 \text { and } s k_{2}[26]=1\right), \\
x^{i+1}[39] \oplus s k_{1}[39]=t^{i+1}[21]\left(\text { since } s k_{2}[15]=1 \text { and } s k_{2}[14]=1\right), \\
x^{i+2}[21] \oplus s k_{1}[21]=t^{i+2}[15]\left(\text { since } s k_{2}[11]=1 \text { and } s k_{2}[10]=0\right), \\
x^{i+3}[15] \oplus s k_{1}[15]=z^{i+3}[45]
\end{gathered}
$$

we can get the equation $x^{i}[45] \oplus s k_{1}[45] \oplus s k_{1}[39] \oplus s k_{1}[21] \oplus s k_{1}[15]=z^{i+3}[45]$ with bias $2^{2} \times\left(-2^{-2}\right) \times\left(-2^{-2}\right) \times\left(-2^{-2}\right)=-2^{-4}$.

Using the correlation of the input and output bits of the active S-boxes in consecutive rounds we give our other main statement for the probability of the differential characteristic shown in Figure 3. 
Theorem 2. Let the key bits of PRINTCIPHER-48 satisfy the following constraints

$$
\begin{gathered}
s k_{2}[30]=0, s k_{2}[29]=1, s k_{2}[28]=1, s k_{2}[21]=0, s k_{2}[20]=1, \\
s k_{1}[46] \oplus s k_{1}[42] \oplus s k_{1}[31]=1, \\
s k_{2}[10]=0, s k_{2}[27]=1, s k_{2}[26]=1, s k_{2}[15]=1, s k_{2}[14]=1, s k_{2}[11]=1, \\
s k_{1}[45] \oplus s k_{1}[39] \oplus s k_{1}[21] \oplus s k_{1}[15]=0 .
\end{gathered}
$$

Then, the probability of the differential characteristic $(100 \ldots 00) \rightarrow(100 \ldots 00) \rightarrow$ $\ldots \rightarrow(100 \ldots 00)$ for $r$ rounds is $2^{-(7.68+1.51 \times(r-4))}$.

Proof. The probability of the first four rounds characteristic is derived as $2^{-7.68}$ in the proof of Theorem 1. For the fifth and later rounds as stated in Lemma 2 $z^{i}[45]$ equals to $x^{i-3}[45] \oplus s k_{1}[45] \oplus s k_{1}[39] \oplus s k_{1}[21] \oplus s k_{1}[15]$ with bias $-2^{-4}$. We know that $x^{i-3}[45]=0$ because the S-box conserves the difference in the leftmost bit for only the input pair $(011,111)$ where the corresponding output pair is $(110,010)$. Since $s k_{1}[45] \oplus s k_{1}[39] \oplus s k_{1}[21] \oplus s k_{1}[15]=0, z^{i}[45]=1$ with bias $2^{-4}$ that is with probability $9 / 16$. Also we know that the probability of being $z^{i}[46]=1$ is 10/16 from the proof of Theorem 11. Thus, for the fifth and later rounds the input pair of the S-box is $(011,111)$ with probability $10 / 16 \times 9 / 16 \approx$ $2^{-1.51}$. As a result, the probability of $r$-round differential characteristic shown

\begin{tabular}{|c|c|c|}
\hline $\begin{array}{c}\text { Combined } \\
\text { Characteristic }\end{array}$ & $\begin{array}{c}\text { Key } \\
\text { Conditions }\end{array}$ & \begin{tabular}{|c|} 
Key \\
Subset
\end{tabular} \\
\hline Characteristic 4 & $\begin{array}{c}s k_{2}[30]=0, s k_{2}[29]=1, s k_{2}[28]=1, s k_{2}[27]=1, s k_{2}[26]=1 \\
s k_{2}[21]=0, s k_{2}[20]=1, s k_{2}[15]=1, s k_{2}[14]=1, s k_{2}[11]=1 \\
s k_{2}[10]=0, s k_{1}[46] \oplus s k_{1}[42] \oplus s k_{1}[31]=1 \\
s k_{1}[45] \oplus s k_{1}[39] \oplus s k_{1}[21] \oplus s k_{1}[15]=0\end{array}$ & $K S^{4}$ \\
\hline Characteristic 5 & $\begin{array}{c}s k_{2}[15]=0, s k_{2}[14]=1, s k_{2}[13]=1, s k_{2}[12]=0, s k_{2}[11]=1 \\
s k_{2}[10]=0, s k_{2}[31]=0, s k_{2}[5] \oplus s k_{2}[4]=0, s k_{2}[27]=1 \\
s k_{2}[26]=1, s k_{1}[22] \oplus s k_{1}[18] \oplus s k_{1}[7]=1 \\
s k_{1}[45] \oplus s k_{1}[39] \oplus s k_{1}[21] \oplus s k_{1}[15]=0\end{array}$ & $K S^{5}$ \\
\hline
\end{tabular}
in Figure 3 is $2^{-(7.68+1.51 \times(r-4))}$.

In Table 4, the key constraints for the combined characteristics are shown.

Table 4. Key constraints for the combined characteristics 4 and 5

Counting the keys in the key space of PRINTCIPHER-48 which satisfies at least one of the conditions in Table 4 we find out that $0.036 \%$ of the key space of the PRINTCIPHER-48 is vulnerable to at least one of the combined characteristics.

\section{Key Recovery}

\subsection{An Attack on 31-Round PRINTCIPHER-48 for $\boldsymbol{K} \boldsymbol{S}^{\mathbf{4}}$}

Assume that PRINTCIPHER-48 uses a key from $K S^{4}$. Therefore the key bits satisfy the conditions: $s k_{2}[30]=0, s k_{2}[29]=1, s k_{2}[28]=1, s k_{2}[27]=1, s k_{2}[26]=1$, 
$s k_{2}[21]=0, s k_{2}[20]=1, s k_{2}[15]=1, s k_{2}[14]=1, s k_{2}[11]=1, s k_{2}[10]=0$, $s k_{1}[46] \oplus s k_{1}[42] \oplus s k_{1}[31]=1$, and $s k_{1}[45] \oplus s k_{1}[39] \oplus s k_{1}[21] \oplus s k_{1}[15]=0$. Using the differential characteristic powered by linear characteristics for 28 rounds in Section 3.3 we have been able to attack on 31-round version of the cipher and recover the key bits $s k_{2}[25-22], s k_{2}[19-16], s k_{1}[47-39]$. For 28 rounds the probability of the differential characteristic is $2^{-43.92}$. The propagation of the active bit in the output of the 28-th round through 3 rounds is shown in Figure 4. In the figure, the difference in the bits in the dotted line is 0 or 1 . We apply the attack using Algorithm 1 .

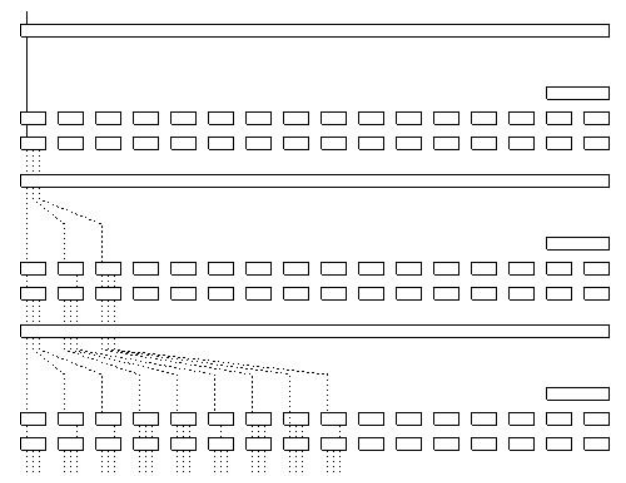

Fig. 4. 3-R attack for the key subset $K S^{4}$

We have calculated the signal to noise ratio as $2^{4.08}$ for the attack on the reduced 31-round PRINTCIPHER-48 using the formula

$$
S / N=\frac{2^{k} \times p}{\alpha \times \beta}=\frac{2^{17} \times 2^{-43.92}}{2^{4} \times 2^{-35}}=2^{4.08}
$$

where $k$ denotes the number of guessed key bits, $p$ is the probability of the characteristic, $\alpha$ is the average count of the subkeys per counted plaintext pairs and $\beta$ is the ratio of the counted pairs to all pairs. Since $S / N$ is bigger than 2 , according to [7], about 4 right pairs are enough to determine the key bits. Thus we need about $4 \times 2^{43.92}=2^{45.92}$ pairs.

The complexity of the attack is as follows. We use $2^{46.92}$ chosen plaintextciphertext data. The number of pairs used in the attack is $2^{45.92} \times 2^{-35}=2^{10.92}$ because of the elimination in step 3 and 5 in the algorithm. Note that in step 4 we make $2^{25,92}$ inverse $S$-box operations. For the counted pairs we make $2^{19.92}$ key dependent permutations guessing the 8 bits $\left(s k_{2}[25-22]\right.$ and $\left.s k_{2}[19-16]\right)$ of the key. We decrease the search space to $2^{15.92}$ using the elimination in step 9 . Then we make $2^{24.92}$ two round decryptions guessing the 9 bits $\left(s k_{1}[47-39]\right)$ of the key. In total, the number of operations in the attack approximately equivalent to $2^{24.92} \times 2=2^{25.92}$ one-round encryptions of PRINTCIPHER- 48 . 


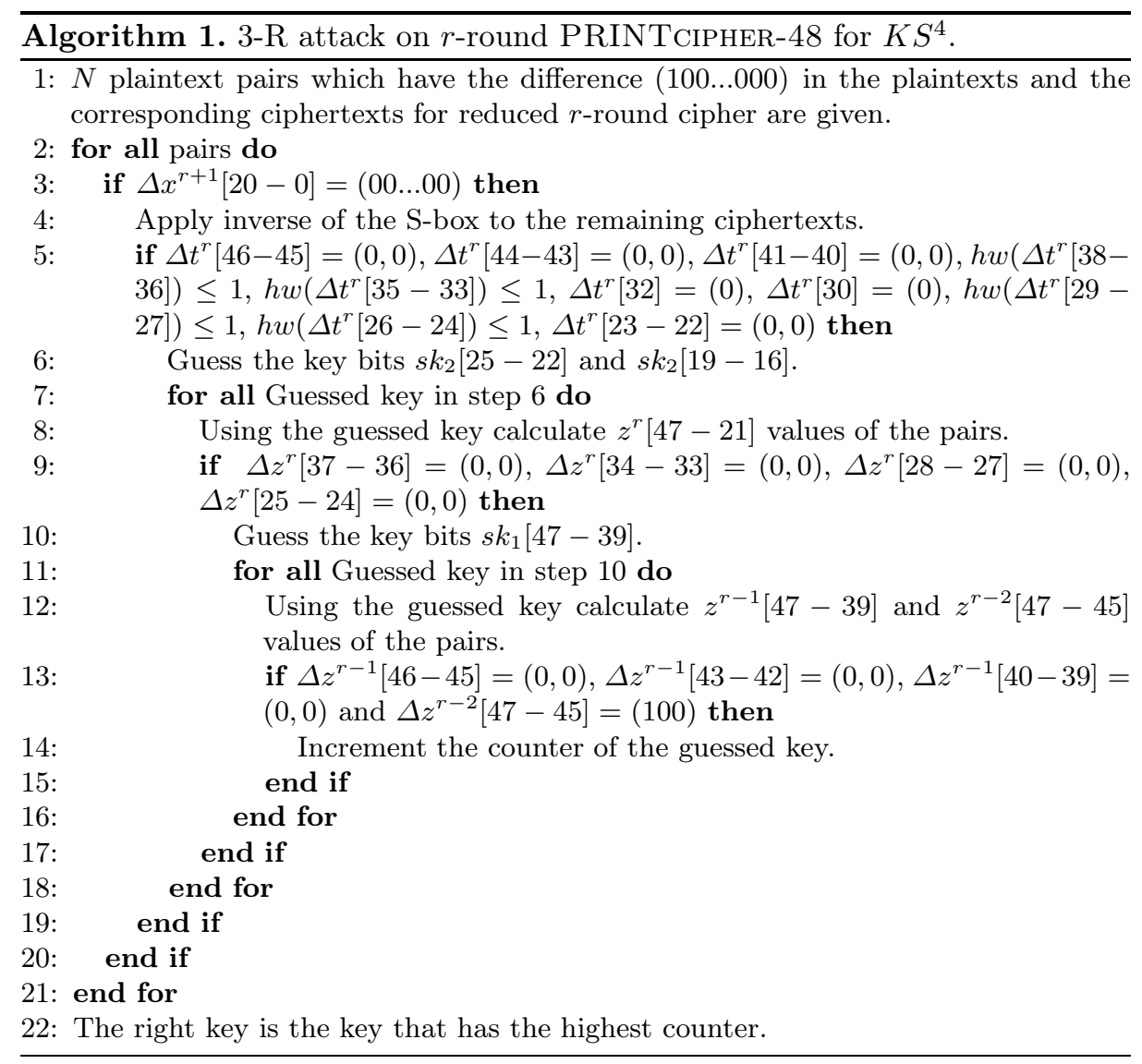

To verify the attack algorithm and the effect of the linear approximations we have implemented the attack for 20-round PRINTCIPHER-48 where the 17round characteristic probability is $2^{-27.31}$ by Theorem 2. We have run Algorithm 1 using $2^{31}$ plaintext-ciphertext data 8 different times. In each of these experiments the correct key is found among the highest counted candidates. If there were no effect of linear approximations on the probability of the differential characteristic, then the probability would be $2^{-34}$ for 17 rounds and $2^{31}$ data would not be sufficient to recover the key bits.

\subsection{An Attack on 29-Round PRINTCIPHER-48 for $\boldsymbol{K} \boldsymbol{S}^{\mathbf{1}}$}

We attack on 29-round PRINTCIPHER-48 for the assumption that the key used in the algorithm is in the key subset $K S^{1}$ using 26-round differential characteristic given in Section 3.2. We can recover the key bits $s k_{2}[27-22], s k_{2}[19-14]$, $s k_{1}[47-39]$ applying the attack. 
The propagation of the active bit in the output of the 26-th round through 3 rounds is shown in Figure 5. We use a similar algorithm as Algorithm 1 to recover the key bits. The differences between the attack algorithms for $K S^{1}$ and $K S^{4}$ are the followings:

- The condition in step 5 will be $\Delta t^{r}[46-45]=(0,0), \Delta t^{r}[44-43]=(0,0)$, $h w\left(\Delta t^{r}[41-39]\right) \leq 1, h w\left(\Delta t^{r}[38-36]\right) \leq 1, h w\left(\Delta t^{r}[35-33]\right) \leq 1, \Delta t^{r}[32]=$ $(0), \Delta t^{r}[30]=(0), h w\left(\Delta t^{r}[29-27]\right) \leq 1, h w\left(\Delta t^{r}[26-24]\right) \leq 1, h w\left(\Delta t^{r}\right.$ $[23-21]) \leq 1$

- The guessed key bits will be $s k_{2}[27-22]$ and $s k_{2}[19-14]$ in step 6 ,

- The condition in step 9 will be $\Delta z^{r}[40-39]=(0,0), \Delta z^{r}[37-36]=(0,0)$, $\Delta z^{r}[34-33]=(0,0), \Delta z^{r}[28-27]=(0,0), \Delta z^{r}[25-24]=(0,0), \Delta z^{r}$ $[22-21]=(0,0)$.

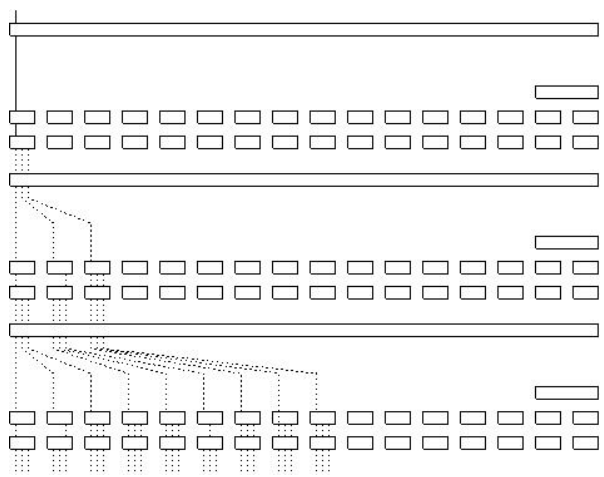

Fig. 5. 3-R attack for the key subset $K S^{1}$

We have calculated the signal to noise ratio as

$$
S / N=\frac{2^{k} \times p}{\alpha \times \beta}=\frac{2^{21} \times 2^{-44.64}}{2^{6} \times 2^{-33}}=2^{3.59} .
$$

About $4 \times 2^{44.64}=2^{46.64}$ pairs are enough to get 4 right pairs.

The complexity of the attack is as follows. We use $2^{47.64}$ chosen plaintextciphertext data. The number of pairs used in the attack is $2^{46.64} \times 2^{-33}=2^{13.64}$ because of the elimination in step 3 and 5 in the algorithm. For the counted pairs we make $2^{26.64}$ key dependent permutations guessing the 12 bits $\left(s k_{2}[27-22]\right.$ and $\left.s k_{2}[19-14]\right)$ of the key. We decrease the search space to $2^{20.64}$ using the elimination in step 9 . Then we make $2^{29.64}$ two round decryptions guessing the 9 bits $\left(s k_{1}[47-39]\right)$ of the key. In total, the number of operations in the attack is approximately equivalent to $2^{30.64}$ one-round encryptions of PRINTCIPHER-48. 


\section{Conclusion}

In this paper, we have used differential and linear cryptanalysis techniques together to analyze the security of PRINTCIPHER. This combined usage is different from differential-linear cryptanalysis [20]. In differential-linear cryptanalysis, a cipher is divided into two parts where differentials and linear approximations are constructed for the first and second parts respectively. In this work, we have used linear approximations to increase the probability of the differentials. Using this method, we have found out that for some of the keys, the probability of an $r$-round differential characteristic is significantly higher than the designers' expected values. With the help of linear approximations we have constructed $r$-round differential characteristics with probability $2^{-(6+1.68 \times(r-3))}$ for $4.54 \%$ of the keys and with probability $2^{-(7.68+1.51 \times(r-4))}$ for $0.036 \%$ of the keys of PRINTCIPHER-48. These observations enable us to develop cryptanalytic attacks on 29 and 31 rounds of PRINTCIPHER- 48 for these key subsets.

Acknowledgments. The authors would like to thank to anonymous reviewers for their valuable comments which helped to improve the quality of this paper.

\section{References}

1. Abdelraheem, M.A., Leander, G., Zenner, E.: Differential Cryptanalysis of RoundReduced PRINTcipher: Computing Roots of Permutations. In: Joux, A. (ed.) FSE 2011. LNCS, vol. 6733, pp. 1-17. Springer, Heidelberg (2011)

2. Aumasson, J.-P., Henzen, L., Meier, W., Naya-Plasencia, M.: QUARK: A lightweight hash. In: Mangard and Standaert [24], pp. 1-15 (2010)

3. Badel, S., Dagtekin, N., Nakahara, J., Ouafi, K., Reffé, N., Sepehrdad, P., Susil, P., Vaudenay, S.: ARMADILLO: A Multi-Purpose Cryptographic Primitive Dedicated to Hardware. In: Mangard and Standaert [24], pp. 398-412 (2010)

4. Biham, E., Biryukov, A., Shamir, A.: Cryptanalysis of Skipjack Reduced to 31 Rounds using Impossible Differentials. In: Stern, J. (ed.) EUROCRYPT 1999. LNCS, vol. 1592, pp. 12-23. Springer, Heidelberg (1999)

5. Biham, E., Dunkelman, O., Keller, N.: Enhancing Differential-Linear Cryptanalysis. In: Zheng, Y. (ed.) ASIACRYPT 2002. LNCS, vol. 2501, pp. 254-266. Springer, Heidelberg (2002)

6. Biham, E., Dunkelman, O., Keller, N.: Differential-Linear Cryptanalysis of Serpent. In: Johansson, T. (ed.) FSE 2003. LNCS, vol. 2887, pp. 9-21. Springer, Heidelberg (2003)

7. Biham, E., Shamir, A.: Differential Cryptanalysis of DES-Like Cryptosystems. In: Menezes, A., Vanstone, S.A. (eds.) CRYPTO 1990. LNCS, vol. 537, pp. 2-21. Springer, Heidelberg (1991)

8. Bogdanov, A., Knudsen, L.R., Leander, G., Paar, C., Poschmann, A., Robshaw, M.J.B., Seurin, Y., Vikkelsoe, C.: Present: An Ultra-Lightweight Block Cipher. In: Paillier, P., Verbauwhede, I. (eds.) CHES 2007. LNCS, vol. 4727, pp. 450-466. Springer, Heidelberg (2007)

9. Bulygin, S.: Algebraic Cryptanalysis of the Round-Reduced and Side Channel Analysis of the Full PRINTcipher-48. Cryptology ePrint Archive, Report 2011/287 (2011), http://eprint.iacr.org/ 
10. De Cannière, C.: TRIvium: A Stream Cipher Construction Inspired by Block Cipher Design Principles. In: Katsikas, S.K., López, J., Backes, M., Gritzalis, S., Preneel, B. (eds.) ISC 2006. LNCS, vol. 4176, pp. 171-186. Springer, Heidelberg (2006)

11. De Cannière, C., Dunkelman, O., Knežević, M.: KATAN and KTANTAN - A Family of Small and Efficient Hardware-Oriented Block Ciphers. In: Clavier, C., Gaj, K. (eds.) CHES 2009. LNCS, vol. 5747, pp. 272-288. Springer, Heidelberg (2009)

12. Cheng, H., Heys, H.M., Wang, C.: PUFFIN: A Novel Compact Block Cipher Targeted to Embedded Digital Systems. In: Fanucci, L. (ed.) DSD, pp. 383-390. IEEE (2008)

13. Daemen, J., Govaerts, R., Vandewalle, J.: Weak Keys for IDEA. In: Stinson, D.R. (ed.) CRYPTO 1993. LNCS, vol. 773, pp. 224-231. Springer, Heidelberg (1994)

14. Dunkelman, O., Indesteege, S., Keller, N.: A Differential-Linear Attack on 12Round Serpent. In: Chowdhury, D.R., Rijmen, V., Das, A. (eds.) INDOCRYPT 2008. LNCS, vol. 5365, pp. 308-321. Springer, Heidelberg (2008)

15. Ågren, M., Johansson, T.: Linear Cryptanalysis of PRINTcipher - Trails and Samples Everywhere. Cryptology ePrint Archive, Report 2011/423 (2011), http://eprint.iacr.org/

16. Hawkes, P.: Differential-Linear Weak Key Classes of IDEA. In: Nyberg, K. (ed.) EUROCRYPT 1998. LNCS, vol. 1403, pp. 112-126. Springer, Heidelberg (1998)

17. Hong, D., Sung, J., Hong, S., Lim, J., Lee, S., Koo, B., Lee, C., Chang, D., Lee, J., Jeong, K., Kim, H., Kim, J., Chee, S.: HIGHT: A New Block Cipher Suitable for Low-Resource Device. In: Goubin, L., Matsui, M. (eds.) CHES 2006. LNCS, vol. 4249, pp. 46-59. Springer, Heidelberg (2006)

18. Izadi, M., Sadeghiyan, B., Sadeghian, S.S., Khanooki, H.A.: MIBS: A New Lightweight Block Cipher. In: Garay, J.A., Miyaji, A., Otsuka, A. (eds.) CANS 2009. LNCS, vol. 5888, pp. 334-348. Springer, Heidelberg (2009)

19. Knudsen, L.R., Leander, G., Poschmann, A., Robshaw, M.J.B.: PRINTcipher: A Block Cipher for IC-Printing. In: Mangard and Standaert [24], pp. 16-32

20. Langford, S.K., Hellman, M.E.: Differential-Linear Cryptanalysis. In: Desmedt, Y.G. (ed.) CRYPTO 1994. LNCS, vol. 839, pp. 17-25. Springer, Heidelberg (1994)

21. Leander, G., Abdelraheem, M.A., AlKhzaimi, H., Zenner, E.: A cryptanalysis of PRINTcipher: The Invariant Subspace Attack. In: Rogaway, P. (ed.) CRYPTO 2011. LNCS, vol. 6841, pp. 206-221. Springer, Heidelberg (2011)

22. Lim, C.H., Korkishko, T.: mCrypton - A Lightweight Block Cipher for Security of Low-Cost RFID Tags and Sensors. In: Song, J.-S., Kwon, T., Yung, M. (eds.) WISA 2005. LNCS, vol. 3786, pp. 243-258. Springer, Heidelberg (2006)

23. Liu, Z., Gu, D., Zhang, J., Li, W.: Differential-Multiple Linear Cryptanalysis. In: Bao, F., Yung, M., Lin, D., Jing, J. (eds.) Inscrypt 2009. LNCS, vol. 6151, pp. 35-49. Springer, Heidelberg (2010)

24. Mangard, S., Standaert, F.-X. (eds.): CHES 2010. LNCS, vol. 6225. Springer, Heidelberg (2010)

25. Matsui, M.: Linear Cryptanalysis Method for DES Cipher. In: Helleseth, T. (ed.) EUROCRYPT 1993. LNCS, vol. 765, pp. 386-397. Springer, Heidelberg (1994)

26. Ohkuma, K.: Weak Keys of Reduced-Round Present for Linear Cryptanalysis. In: Jacobson Jr., M.J., Rijmen, V., Safavi-Naini, R. (eds.) SAC 2009. LNCS, vol. 5867, pp. 249-265. Springer, Heidelberg (2009)

27. Ojha, S.K., Kumar, N., Jain, K., Sangeeta, L.: TWIS - A Lightweight Block Cipher. In: Prakash, A., Sen Gupta, I. (eds.) ICISS 2009. LNCS, vol. 5905, pp. 280-291. Springer, Heidelberg (2009) 
28. Standaert, F.-X., Piret, G., Gershenfeld, N., Quisquater, J.-J.: SEA: A Scalable Encryption Algorithm for Small Embedded Applications. In: Domingo-Ferrer, J., Posegga, J., Schreckling, D. (eds.) CARDIS 2006. LNCS, vol. 3928, pp. 222-236. Springer, Heidelberg (2006)

29. Sun, X., Lai, X.: The Key-Dependent Attack on Block Ciphers. In: Matsui, M. (ed.) ASIACRYPT 2009. LNCS, vol. 5912, pp. 19-36. Springer, Heidelberg (2009)

30. Wagner, D.: The Boomerang Attack. In: Knudsen, L.R. (ed.) FSE 1999. LNCS, vol. 1636, pp. 156-170. Springer, Heidelberg (1999)

31. Guo, S.-Z., Zhao, X.-J., Wang, T.: Fault-Propagation Pattern Based Dfa on Spn Structure Block Ciphers using Bitwise Permutation, with Application to Present and PRINTcipher. Cryptology ePrint Archive, Report 2011/086 (2011), http://eprint.iacr.org/

32. Zhang, W., Zhang, L., Wu, W., Feng, D.: Related-Key Differential-Linear Attacks on Reduced AES-192. In: Srinathan, K., Rangan, C.P., Yung, M. (eds.) INDOCRYPT 2007. LNCS, vol. 4859, pp. 73-85. Springer, Heidelberg (2007)

\section{A Characteristics for $4.54 \%$ of the Keys of PRINTCIPHER-48}

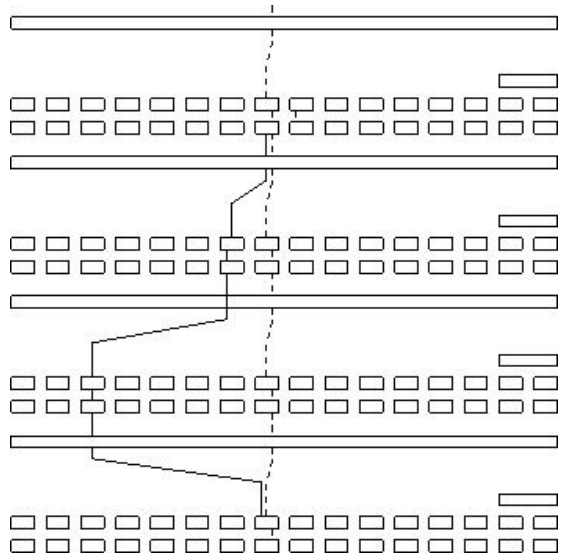

Fig. 6. Combined characteristic 2 


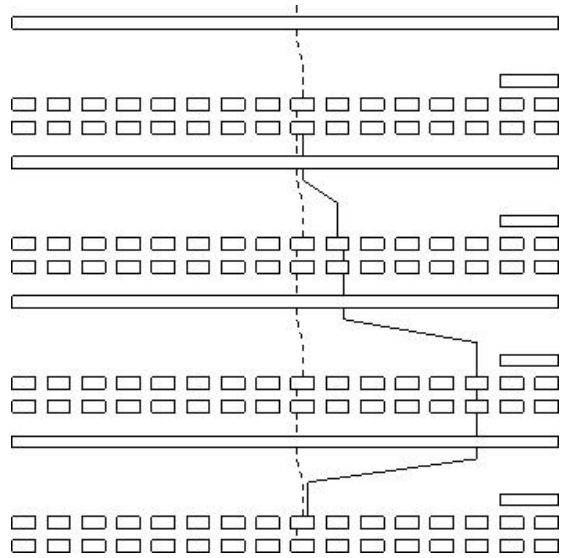

Fig. 7. Combined characteristic 3

\section{B Characteristic for $\mathbf{0 . 0 3 6 \%}$ of the Keys of PRINTCIPHER-48}

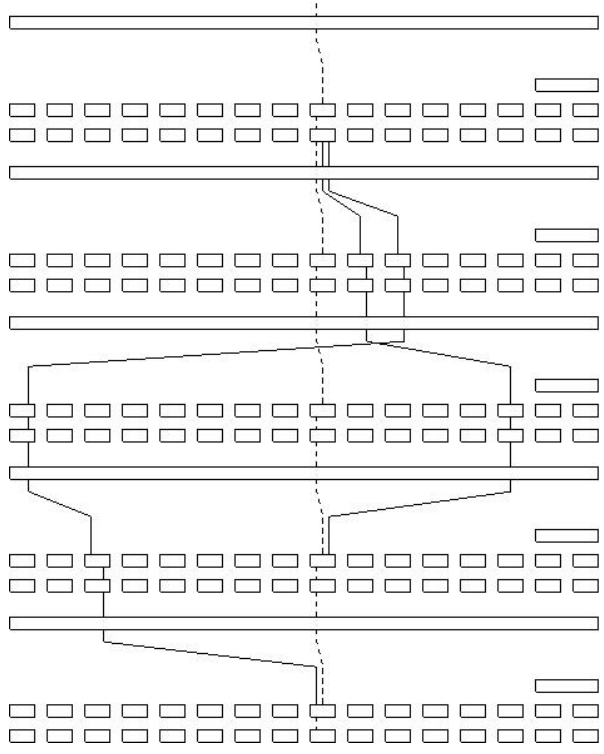

Fig. 8. Combined characteristic 5 